\title{
Abraham Maslow, las nececidades humanas y su relación con los cuidadores profesionales
}

\author{
Abraham Maslow, human needs and their \\ relationship with professional caregivers
}

Abraham Maslow, necessidades humanas e sua relação com cuidadores profissionais

\author{
Francisco-Javier Castro-Molina
}

\begin{abstract}
Escuela Universitaria de Enfermería de "Nuestra Señora de Candelaria" (adscrita a la Universidad de La Laguna-ULL). Asociación Canaria de Historia de la Profesión Enfermera (ACHPE)

Cómo citar este artículo en edición digital: Castro-Molina, F.J. (2018). Abraham Maslow, las necesidades humanas y su relación con los cuidadores profesionales. Cultura de los Cuidados (Edición digital), 22(52). Recuperado de http://dx.doi.org/10.14198/cuid.2018.52.09
\end{abstract}

Correspondencia: Asociación Canaria de Historia de la Profesión Enfermera (ACHPE), calle San Martín 62, 38001-Santa Cruz de Tenerife

Correo electrónico: fcastrom@ull.edu.es; fjcastrom7@gmail.com Recibido: 27/03/2018; Aceptado: 11/5/2018

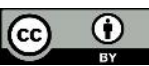

\section{ABSTRACT}

Introduction: The Humanistic Psychology was the result of a process generated from the first national meeting, celebrated in 1962, of the Americam Association for Humanistic Psycology (AAHP) organized by Abraham Maslow, among others. This denomination, the one of Humanist Psychology, was the product of a heated controversy that shuffled very diverse names.

Objective: Establish a clear relationship between the theoretical bases of Humanistic Psychology and those proposed by Nursing.

Method: There was a "thorough examination" of each of the theories that define the different currents of thought.

Results: But Has it historically contributed to the development of health science professions such as medicine or nursing? From the first moments, the conformation of the theoretical body of the Nursing, relied on the postulates established by Maslow, Dorsch and Rulla. The concept of basic human need in uniting with the theoretical models of Orem, Watson, King, Abdellah, Henderson, Levine, Roy, and Gordon identified a series of clearly defined needs. Vera Fry goes further, establishing the concept of nursing diagnosis, setting five areas of needs that allow to state the diagnosis.

Keywords: Maslow, human needs, nursing care, Nursing, Humanistic Psychology.

\section{RESUMO}

Introdução: A Psicologia Humanista foi o resultado de um processo gerado a partir do primeiro encontro nacional, celebrado em 1962, da Associação Americana de Psicologia Humanista (AAHP), organizada 
por Abraham Maslow, entre outros. Essa denominação, a da Psicologia Humanista, foi o produto de uma controvérsia acalorada que embaralhou nomes muito diversos.

Objetivo: Estabeleça uma relação clara entre as bases teóricas da Psicologia Humanista e as propostas pela Enfermagem.

Método: Houve um "exame minucioso" de cada uma das teorias que definem as diferentes correntes do pensamento.

Resultados: Mas Tem historicamente contribuído para o desenvolvimento de profissões de ciências da saúde, como medicina ou enfermagem? Desde os primeiros momentos, a conformação do corpo teórico da Enfermagem, baseou-se nos postulados estabelecidos por Maslow, Dorsch e Rulla. O conceito de necessidade humana básica em unir-se com os modelos teóricos de Orem, Watson, King, Abdellah, Henderson, Levine, Roy e Gordon identificou uma série de necessidades claramente definidas. Vera Fry vai além, estabelecendo o conceito de diagnóstico de enfermagem, estabelecendo cinco áreas de necessidades que permitem indicar o diagnóstico.

Palavras chave: Maslow, necessidades humanas, cuidados de enfermagem, Enfermagem, Psicologia Humanista.

\section{RESUMEN}

Introducción: La Psicología Humanística fue el resultado de un proceso generado a partir del primer encuentro nacional, celebrado en 1962, de la Americam Association for Humanistic Psycology (AAHP) organizado por Abraham Maslow, entre otros. Esta denominación, la de Psicología Humanista, fue el producto de una acalorada polémica que barajó nombres muy diversos.

Objetivo: Establecer una relación clara entre las bases teóricas de la Psicología
Humanística y las propuestas por la Enfermería.

Método: Se realizó un "examen exhaustivo" de cada una de las teorías que definen las diferentes corrientes del pensamiento.

Resultados: Pero, ¿ha contribuido históricamente al desarrollo de las profesiones de las Ciencias de la Salud tales como la Medicina o la Enfermería? Desde los primeros momentos, la conformación del cuerpo teórico de la Enfermería, se apoyó en los postulados establecidos por Maslow, Dorsch y Rulla. El concepto de la necesidad humana básica al unirse con los modelos teóricos de Orem, Watson, King, Abdellah, Henderson, Levine, Roy y Gordon identificó una serie de necesidades claramente definidas. Vera Fry va más allá, estableciendo el concepto de diagnóstico enfermero, fijando cinco áreas de necesidades que permiten enunciar el diagnóstico.

Palabras clave: Maslow, necesidades humanas, cuidados enfermeros, Enfermería, Psicología Humanista.

\section{INTRODUCCIÓN}

La aparición histórica de la Psicología Humanística fue el producto de una larga gestación materializada en el primer encuentro Nacional de la Americam Association for Humanistic Psycology (AAHP) celebrado en 1962. Abraham Maslow, junto a Anthony Sutich, Joe Adams, Dorothy Lee y el propio Clark Moustakas son los que conformaron el comité organizador (Dorsch, 2002). Esta denominación, la de Psicología Humanista, fue el producto de una acalorada polémica que barajó nombres como Ontopsychology, Axiopsychology, Metapsychology, Self 
Psychology, Autonomous Psychology, Selfdirective Psychology y Person Psychology. Finalmente se llegó al acuerdo, no unánime, de sustituirlo por el de American Association for Humanistic Psychology. A ello se agregó la decisión relativa al título definitivo que debería llevar la revista de la Asociación. Fundada por Abraham Maslow y Anthony Sutich en 1958, no publicó su primer número hasta 1961. El comité de redacción presentó serias dificultades en encontrar un título que se ajustara al mensaje que se quería enviar. The Journal of Self Psychology era el propuesto inicialmente en consonancia con el título del libro The Self, editado por Clark Moustakas en 1956. En una carta del 29 de diciembre de 1959, dirigida a Anthony Sutich, Stephen Cohen propuso el título de The Journal of Humanistic Psychology (Maslow, 1991; Max-Neef, 1998). Maslow y su teoría de las necesidades, como ya se ha puntualizado, fue una de las precursoras de la Psicología Humanista a la que definió la Tercera Fuerza. A la cola de la teoría formulada por Sigmund Freud y la corriente conductista, ésta se convirtió en trascendental en el enfoque de las necesidades (Blanco, 2002; Maslow, 1991).

\section{MÉTODO}

El proceso de investigación del presente estudio constó de dos fases: primeramente se procedió a una revisión bibliográfica de las publicaciones existentes en lengua española desde tres ópticas diferentes claramente definidas: Maslow y la Psicología Humanística frente a la Enfermería, su teóricas y sus teorías (diversos trabajos de investigación publicados y bibliografía existente relacionada con el tema). Finalmente, se realizó una búsqueda en los diferentes recursos electrónico y bases de datos
(CUIDEN, SCIELO y PubMed) atendiendo a las palabras clave citadas con anterioridad.

\section{RESULTADOS}

En los albores de su carrera profesional, Maslow descubrió, mientras trabajaba con monos, que ciertas necesidades prevalecen sobre otras. Para él, tanto las necesidades humanas como la forma de ser satisfechas presentaban relevancias semejantes, conformándose así la experiencia humana. Además de considerar las evidentes aguaaire-comida-sexo, el autor lo amplió a cinco grandes bloques: las necesidades fisiológicas, las necesidades de seguridadreaseguramiento, la necesidad de amorpertenencia, la necesidad de estima y la necesidad de actualizar el sí mismo (self), siguiendo este orden (Maslow, 1991). Las necesidades fisiológicas, entre las que se encuentran las relacionadas con la supervivencia del individuo, es decir, las primordiales, básicas, esenciales, elementales, son aquellas que el ser humano necesita para sobrevivir. Entre ellas destacan la de alimentación, la de abrigo, la de deseo sexual, la de respiración, la de reproducción, la de descanso-sueño, la de acariciar-amar, la del comportamiento maternal, la de mantenimiento de la temperatura corporal, la de homeostasis, y la de alivio de dolor, entre otras. Estas necesidades pueden definirse a partir de tres características principales: origen somático, independencia relativa y potencia. El primero, el origen somático, va en concordancia con el adjetivo «fisiológicas». Éstas tienen un origen corporal, y en este sentido se diferencian del resto de las necesidades. En algunos casos, como en los clásicos ejemplos del hambre, la sed y el deseo sexual, hay una base somática localizada específicamente en ciertas partes del cuerpo, situación que no está presente en 
otros casos como la necesidad de descanso, de sueño o las conductas maternales. Cuando abordamos la independencia relativa encontramos que las necesidades lo son entre sí en relación a otras necesidades no fisiológicas. Así, queda claro que el hambre, la sed y el deseo sexual, por ejemplo, se satisfacen independientemente la una de la otra. Por último, la potencia, en la que unas necesidades tienen una mayor fuerza con respecto al resto. Es decir, si una persona carece de alimento, seguridad, amor y estima, probablemente sentirá con mayor fuerza la ausencia del alimento antes que otra cosa, y por tanto intentará satisfacer en primer lugar la necesidad fisiológica por ser éstas las más potentes. Así, si todas las necesidades no están satisfechas, el organismo se encontrará dominado por las necesidades ubicadas en la base de la pirámide, es decir, las fisiológicas, mientras que las otras quedarán desplazadas a un segundo plano (Max-Neef, 1998).

Por encima de las necesidades fisiológicas encontramos las de seguridad, particularmente importante para los niños. La naturaleza del hombre precisa de protección frente al peligro en un ambiente agradable, previendo cualquier situación. A ella se unen las necesidades de estabilidad, de ausencia de miedo-ansiedad a lo desconocido, al caos o a la confusión, a perder el control de sus vidas, de ser vulnerables o débiles a circunstancias nuevas. Estas necesidades adquieren en los niños la forma de búsqueda de la rutina, de lo previsible, mientras que en los adultos se materializa en la búsqueda de la estabilidad económico-laboral.

Superpuestas, las necesidades sociales y las de estima. Las primeras son las que algunos autores han denominado de amor, de pertenencia, de afecto. Éstas están vinculadas a las relaciones interpersonales $\mathrm{o}$ de interacción social, verdaderos motivadores activos de la conducta. Las segundas, las de estima o también llamadas de ego o de reconocimiento, incluyen la preocupación de la persona por alcanzar la maestría, la competencia, el estatus. Maslow agrupa estas necesidades en dos clases, las referidas al amor propio, al respeto a sí mismo, a la estimación propia y la autoevaluación, y las que aluden a los otros, las necesidades de reputación, condición, éxito social y de reconocimiento por sus iguales. La satisfacción de estas necesidades proviene al individuo de sentimientos de autoconfianza, de suficiencia, de utilidad, en contraposición a la frustración, situación generadora de sentimientos de inferioridad, debilidad y desamparo (Maslow, 1991).

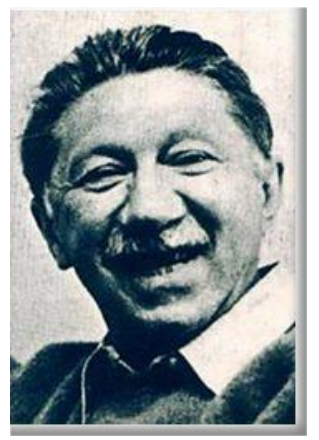

IMAGEN 1. Abraham Harold Maslow (1908-1970)

Por último, las necesidades de autorrealización, también conocidas como de autosuperación. Para Maslow, la autorrealización es un ideal al que todo hombre desea llegar, satisfaciéndose mediante oportunidades para desarrollar el talento y su potencial al máximo, expresando ideas y conocimientos, creciendo y desarrollándose como una gran persona. En este contexto, el individuo precisa de trascender creando su propia obra. Los que la logran son los que el autor denomina «seres íntegros».

En 1971, tras una exhaustiva revisión de su 
propuesta, agregó a su jerarquía de necesidades dos clases más: la necesidad de saber-comprender y las necesidades estéticas. La primera o necesidades de orden cognoscitivo no las ubicó en un lugar concreto dentro de las categorías preestablecidas. Se basó en la necesidad que tiene el hombre por saber y descubrir cosas conocidas y desconocidas. Las segundas están relacionadas con el deseo del orden y de la belleza, tanto de lo que lo rodea como de sí mismo.

Esta teoría tuvo importantes defensores como también destacados detractores. En contraposición, Manfred Max-Neef estableció el problema de la piramidalidad social, llevando la jerarquización de las necesidades a la estructura social. En su obra Desarrollo a escala humana planteó que las necesidades son universables, finitas $\mathrm{y}$ pocas, además de clasificables. A Max-Neef se le unió la postura de Wahba y Bridley, en su obra Maslow Reconsidered: A review of Research on te Need Hierarchy Theory (1976). Encontraron que existían pocas evidencias de que, como apuntaba Maslow, las necesidades precisan organizarse de manera jerárquica con un considerable orden.

Tanto Dorsch como Rulla procuran aplicar la Tercera Fuerza a la profesión de los cuidadores profesionales. El primero definió las necesidades como la expresión de lo que un ser vivo requiere indispensablemente para su conservación y desarrollo, es decir, es el sentimiento ligado a la vivencia de una carencia, asociada a un esfuerzo orientado a suplir faltas, a satisfacer la tendencia y a corregir las carencias. El segundo las determinó como tendencias innatas a la acción que derivan de un déficit del organismo o de potencialidades naturales inherentes al hombre que buscan ejercicio y actualización (Max-Neef, 1998).

\section{DISCUSIÓN-CONCLUSIÓN}

Pero, ¿qué influencia ha tenido esta corriente del pensamiento en otras disciplinas?, ¿ha contribuido históricamente al desarrollo de las profesiones de las Ciencias de la Salud tales como la Medicina o la Enfermería? Desde los primeros momentos, la conformación del cuerpo teórico de la Enfermería, se apoyó en los postulados establecidos por Maslow, Dorsch y Rulla. El concepto de la necesidad humana básica al unirse con los modelos teóricos de Orem, Watson, King, Abdellah, Henderson, Levine, Roy y Gordon identificó una serie de necesidades presentes: Respiración, Mantenimiento de la energía, Movimiento-actividad, Descanso-sueño, Eliminación, Seguridad y Comunicación e interacción (Roy \& Andrews, 1990; Marriner Tomey \& Raile Alligood, 2007). Vera Fry va más allá, estableciendo el concepto de diagnóstico enfermero, fijando cinco áreas de necesidades sobre las que es posible enunciar el diagnóstico: Tratamientomedicación, Higiene personal, Necesidades ambientales, Guía-enseñanza, y Necesidades humanas o personales. Empleemos una clasificación $u$ otra, si queda claro que el enfoque que delimita al problema debe girar en torno a la perspectiva humanista establecida, desde 2008, por la Organización Mundial de la Salud (Henderson, 1971).

Cuando cartografiamos la evolución de la enfermería encontramos que Kérouac, Pepin, Ducharme, Duquette y Major (1996) sugieren que el evolución histórico de la de la disciplina de los cuidadores profesionales puede ser entendido como una progresión de paradigmas, de la categorización, donde se describe una fase de orientación hacia la salud pública (1850-1900) y una fase de orientación hacia la enfermedad (1900-1950), en la que se establece una delegación de la 
práctica médica en la enfermera. A él le sigue el paradigma de la integración, en el que el concepto de persona ocupa un lugar central, gracias a la Psicología Humanística. Finalmente, el paradigma de la integración (último cuarto del siglo XX), que presenta una marcada sensibilidad se ve contaminada por la antropología cultural (Sellán, 2006).

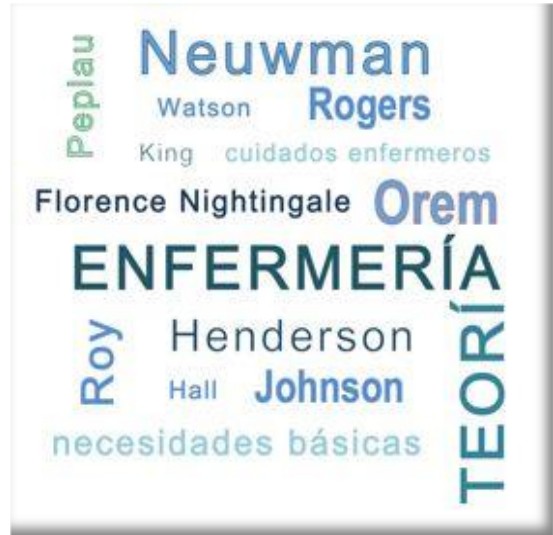

IMAGEN 2. El edificio de las teorías enfermeras [Foto: Francisco J. Castro]

$\mathrm{Al}$ centrar nuestra atención en los trabajos de Peplau, Roy y Watson comprobamos que no existe un reconocimiento por parte de las autoras de las ideas de Maslow. Mientras Watson formula hasta 22 citas en sus textos en referencia a la teoría de las necesidades de Maslow, Peplau y Roy, 3 y 2 respectivamente. En el texto de Watson Nursing: The philosophy and science of caring, dedica la totalidad de la segunda parte de esta obra a la asistencia para la satisfacción de las necesidades humanas, integrando la pirámide de Maslow en su propuesta para definir las necesidades humanas. Las divide en necesidades de orden inferior (biofísicas y psicofísicas), necesidades de orden superior (psicosociales) y necesidades de orden máximo (intrapersonales) a las que también definió como auto-realización (Watson, 1985; Watson, 2005; Peplau, 1993).
En contraposición, en autoras como Orem y Henderson es grosera esta influencia. La primera, pese a no reconocer la influencia maslowiana, establece una influencia de las teorías psicológicas humanísticas (Orem, 1993). Este influjo queda patente al observar la gráfica de jerarquización que propone Maslow: el mantenimiento de un aporte de aire suficiente, el mantenimiento de un aporte de agua suficiente, el mantenimiento de un aporte de alimentos suficiente, la provisión de cuidados asociados con los procesos de eliminación y los excrementos, el mantenimiento del equilibrio entre la actividad y el reposo, el mantenimiento del equilibrio entre la soledad e interacción social, la prevención de peligros para la vida, el funcionamiento y el bienestar humanos y la promoción del funcionamiento y desarrollo humanos dentro de los grupos sociales de acuerdo con el potencial humano, el conocimiento de las limitaciones humanas y el deseo humano de ser normal.

Con respecto al segundo planteamiento, Henderson se ve influenciada por la idea holística del individuo en la que se aúnan diferentes esferas: la física, la psíquica y la social. Concibe una serie de necesidades que las distribuye en 14 categorías: respirar normalmente, comer y beber de forma adecuada, evacuar los desechos corporales, moverse y mantener una postura adecuada, dormir y descansar, elegir la ropa adecuada, mantener la temperatura del cuerpo dentro de un margen adecuado seleccionando la ropa y modificando las condiciones ambientales, mantener la higiene corporal y un buen aspecto y proteger la piel, evitar los peligros del entorno y evitar dañar a los demás, comunicarse con los otros expresando las propias emociones, necesidades, temores y opiniones, actuar con arreglo a la propia fe, actuar de manera 
que se tenga la sensación de satisfacción con uno mismo, disfrutar o participar en diversas formas de entretenimiento $y$ aprender, descubrir o satisfacer la curiosidad para alcanzar un desarrollo y una salud normales y acudir a los centros sanitarios disponibles. Además integra el concepto de dependencia e independencia que Maslow desarrolla en 1991, entendiendo al individuo independiente como aquel que tiene todas sus necesidades cubiertas (García \& Sellán, 1995; Sellán \& Blanco, 2005; Vázquez, 2006).

Hasta la fecha no existe ningún estudio serio que aporte una visión histórica de la influencia que ha supuesto la propuesta de Maslow en la conformación de la base teórica de la profesionalización de los cuidadores. Con el presente trabajo se ha buscado establecer esa interrelación e influjo que históricamente se desarrolló desde la Psicología Humanística, convirtiéndose en un verdadero acicate en el andamiaje que se precisó para la conformación del marco teórico disciplinar de la profesión enfermera (Kérouac et al., 1996).

\section{BIBLIOGRAFÍA}

- Blanco, F. (2002). El cultivo de la mente. Madrid: Machado.

- Dorsch, F. (2002). Diccionario de psicología (8. a ed.). Barcelona: Herder.

- García, C. y Sellán, MC. (1995). Fundamentos teóricos y metodológicos de enfermería. Madrid: Lebosend.

- Henderson, V. (1971). Principios Básicos de los Cuidados de Enfermería. Ginebra: CIE.

- Kérouac, S.; Pepin, J.; Ducharme, F.; Duquette, A. y Major, F. (1996). El pensamiento enfermero. Barcelona: Masson.

- Marriner Tomey, A. Raile Alligood, M. (2007). Modelos y teorías en enfermería (6. ed.). Madrid: Elsevier Mosby.

- Maslow, A. (1991). El Hombre Autorrealizado: Hacia una Psicología del Ser (9. a ed.). Barcelona: Kairós.

- Maslow, A. (1991). Motivación y personalidad. Madrid: Ediciones Díaz de Santos.

- Max-Neef, M. (1998). Desarrollo a escala humana. Conceptos, aplicaciones y algunas reflexiones (2.․ ed.). Barcelona: Icaria.

- Orem, D. (1993). Modelo de Orem. Conceptos de enfermería en la práctica. Barcelona: Ediciones Científicas y Técnicas.

- Peplau, H. (1993). Relaciones interpersonales en enfermería: un marco de referencia para la enfermería psicodinámica. Barcelona: Ediciones Científicas y Técnicas.

- Roy, C. y Andrews, H. (1990). The Roy adaptation model (2.. ${ }^{\mathrm{a}}$ ed.). Stamford: Appleton \& Lange.

- Sellán, MC. (2006). La Psicologización del Cuidado: Krausismo, Psicología y Enfermería. Revista de Historia de la Psicología, 27(2-3), 127-133.

- Sellán, MC. y Blanco, F. (2005). Hacia un modelo historiográfico sobre las relaciones entre enfermería y psicología. Revista de Historia de la Psicología, 26(23), 213-219.

- Vázquez, A. (2006). Enfermería y Psicología: Una historia conjunta. Póster presentado al XIX Symposium de la SEHP, 27-29 de Abril 2006 Miraflores de la Sierra Madrid.

- Watson, J. (1985). Nursing: The philosophy and science of caring. Boulder, CO.: Colorado Associated University Press.

- Watson, J. (2005). Caring Science as Sacred Science. Philadelphia: FA Davis. 